\title{
Overexpression of MUC16 predicts favourable prognosis in MUC16-mutant cervical cancer related to immune response
}

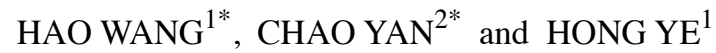 \\ ${ }^{1}$ Department of Gynaecology and Obstetrics, The First College of Clinical Medical Science, China Three Gorges University; \\ ${ }^{2}$ Department of Orthopaedics, The People's Hospital of China Three Gorges University, Yichang, Hubei 443000, P.R. China
}

Received September 5, 2019; Accepted April 29, 2020

DOI: $10.3892 /$ etm.2020.8836

\begin{abstract}
Cervical cancer (CC) is the fourth ranking gynaecologic tumour in women worldwide, with respect to both incidence and mortality. MUC16 is one of the most frequently mutated genes, which functions as a tumour marker in CC. In the present study, mutation, clinical and RNA-Seq data collected from The Cancer Genome Atlas database were used to investigate the association between MUC16 mutation, immune response and clinical prognosis in CC. mRNA expression levels from the TCGA datasets and the results from the present study demonstrated that MUC16 was overexpressed in CC samples; however, there was no difference between mutant and wild-type $\mathrm{CC}$ samples. Furthermore, the results indicated that patients with MUC16-mutant overexpression had a prolonged survival time. In addition, overexpression of MUC16 was associated with immune responses in the microenvironment of MUC16-mutant CC. Immune responses were upregulated in patients with early-stage MUC16-mutant. The results from the present study provided novel biomarkers for potential immunotherapy approaches for CC.
\end{abstract}

\section{Introduction}

In 2018, it was estimated that there were 570,000 new cases and 311,000 CC-associated mortality cases (1). Furthermore, $\sim 90 \%$ of cases occur in low-income and middle-income countries, where there is a lack of organized screening (2). CC is preventable to a large extent, and $\mathrm{CC}$ at the early stage can be treated with surgery or radiation (3). Cervical squamous cell carcinoma (CSCC) accounts for $\sim 70-80 \%$ of CC and $20-25 \%$ of endocervical adenocarcinomas (EACs). EAC is

Correspondence to: Professor Hong Ye, Department of Gynaecology and Obstetrics, The First College of Clinical Medical Science, China Three Gorges University, 183 Yiling Street, Yichang, Hubei 443000, P.R. China

E-mail: yehongyehong998@126.com

${ }^{*}$ Contributed equally

Key words: cervical cancer, cervical squamous cell carcinoma, MUC16, mutation, immune response commonly associated with worse clinical outcome and prognosis compared with CSCC (4). Traditionally, clinical stage is considered the most prominent prognostic parameter, which determines the modality adopted for treatment to a great extent. CSCC antigen levels are closely associated with CC during the early stage (5). Therefore, research on early-stage $\mathrm{CSCC}$ is essential for accurate diagnosis and effective treatment of CC.

Synthesis of mucin (MUC) is essential for the formation of the mucous barrier, which helps protect the epithelia of most organs, such as the stomach, from physical and chemical damages and infection (6,7). Mucins are generally large high-molecular-weight glycoproteins that are classified into two subgroups, the secreted mucins and the membrane-bound mucins (6,7). MUC2, MUC5AC, MUC5B, MUC6, MUC7, MUC9 and MUC19 are secreted mucins, whereas MUC3A/B, MUC4, MUC12, MUC13, MUC15, MUC16, MUC17, MUC20 and MUC21 are membrane-bound mucins. These mucins are considered to play important roles in cellular interactions, molecular cell signalling and biological processes (8). Furthermore, it has been demonstrated that dysregulation of these mucins occurs in different types of cancer. For example, MUC1 is overexpressed in pancreatic, lung, breast, colon, ovarian and prostate cancers; MUC4 is overexpressed in colon adenocarcinoma and pancreatic cancer; and MUC16 is elevated in ovarian and pancreatic cancers (8).

CA125 was first identified $\sim 38$ years ago in a screening of antibodies against ovarian cancer antigens. After two decades, it was cloned and characterized as a membrane-bound mucin named MUC16 (9). MUC16 is the largest membrane-bound mucin and represents an adverse prognostic marker of human cancer. Previous studies have demonstrated that MUC16 is overexpressed in several types of cancer, including breast, lung, pancreatic and colorectal cancers $(10,11)$. Increasing evidence suggested that MUC16 is associated with immune responses in human cancer. Gubbels et al (12) reported that ovarian tumour cells with high levels of MUC16 are unable to be attacked by natural killer cells and monocytes. Patankar et al (13) demonstrated that tumour-derived MUC16 functions as a suppressor of the immune response that is directed against ovarian tumours. Furthermore, Fan et al (14) reported that the MUC16 C terminus promotes forkhead box P3 expression and enrichment of tumour-associated regulatory cells in tumour tissues, through tumour-secreted IL-6 activation of the Janus 
kinase 2/signal transducer and activator of transcription 3 signalling pathway in pancreatic cancer. Recent studies have demonstrated that MUC16 mutations are associated with better survival outcomes and immune responses in gastric and endometrial cancers $(15,16)$. Furthermore, MUC16 has been indicated to serve as a tumour marker in different types of gynaecological cancer, including CC (17). Although MUC16 is regarded as one of the most frequently mutated genes in $\mathrm{CC}$, the associations between MUC16 mutations, immune responses and clinical prognosis remain unclear. Subsequently, the present study used mutation, clinical and RNA-Seq data collected from The Cancer Genome Atlas (TCGA) database (https://portal.gdc.cancer.gov), in order to investigate the association between MUC16 mutation and immune responses, as well as clinical prognosis in CC.

\section{Materials and methods}

Raw data. Data associated with mutation, clinical parameters, copy number variation (CNV), DNA methylation and RNA-Seq of CC samples were downloaded from the TCGA database. MUC16 RNA-Seq data from the various types of cancer were downloaded from the TCGA database (https://portal.gdc. cancer.gov/). The RNA-Seq data were presented in terms of fragments per kilobase million (FPKM). Furthermore, the GSE9750 dataset was downloaded from the Gene Expression Omnibus (GEO) database (https://www.ncbi.nlm.nih.gov/ geo/query/acc.cgi?acc=GSE9750) $(18,19)$. MUC16 expression was assessed in $286 \mathrm{CC}$ and 240 CSCC clinical samples $(\leq 4,000$ days of follow-up data) from the TCGA datasets. Data used in TCGA CNV, DNA methylation and clinical data analyses were matched with the respective expression data.

Definitions of clinical survival and recurrence types. Three types of clinical survival and recurrence outcomes were selected in the present study: Overall survival (OS), disease-specific survival (DSS) and progression-free survival (PFS). The outcomes were defined as follows: OS referred to the period of time from the date of diagnosis to the date of mortality from any cause; DSS referred to the period of time from the date of initial diagnosis to the date of last contact or the date of mortality from another cause; and PFS referred to the period from the date of diagnosis to the date of new tumour occurrence (20).

Patient information and tissue collection. CC tissues and adjacent normal tissues were obtained from 9 patients; 3 patients used to detect the MUC16 protein expression levels between adjacent normal tissue and $\mathrm{CC}$ tissue, 3 patients used to detect the MUC16 protein expression levels in wild-type CC tissues; and 3 patients used to detect the MUC16 protein expression levels in mutant type CC tissues (age range, 44-51 years; median age, 47 years); who underwent radical resection at The First College of Clinical Medical Science, China Three Gorges University (Yichang, China) between March 2019 and July 2019. All samples were stored at $-80^{\circ} \mathrm{C}$. The inclusion criteria were as follows: i) All patients were diagnosed with $\mathrm{CC}$, following colposcopy and cervical tissue biopsy; ii) no chemotherapy or radiotherapy was performed prior to surgery, and iii) all patients had complete clinical data. Exclusion criteria: i) Patients with incomplete clinical data; and ii) patients who refused to participate in this study. All experimental procedures were approved by the Ethics Committee of The First College of Clinical Medical Science, China Three Gorges University (Yichang, China). Written informed consent was provided by all patients prior to the study.

Western blotting. Total protein was extracted from CC tissues and adjacent normal tissues using Cell lysis buffer (cat. no. P0013; Beyotime Institute of Biotechnology, China), and the protein concentration was quantified using a BCA Assay kit (cat. no. P0012S; Beyotime Institute of Biotechnology, China). A total of $50 \mu \mathrm{g}$ protein was added to each sample well, separated by $5 \%$ SDS-PAGE prior to being transferred onto polyvinylidene fluoride membranes. Dried, non-fat milk powder was used for blocking, for $1 \mathrm{~h}$ at room temperature. The membranes were incubated with a MUC16 monoclonal antibody (cat. no. BM5743; 1:2,000; Wuhan Boster Biological Technology, Ltd.) at $4^{\circ} \mathrm{C}$ for $12 \mathrm{~h}$, prior to incubation with a horseradish peroxidase (HRP)-labelled secondary antibody (cat. no. BM2006, 1:1,000; Wuhan Boster Biological Technology, Ltd.) at room temperature for $1 \mathrm{~h}$. ECL luminous liquid A and B (cat. no. P0018S, Beyotime Institute of Biotechnology) were mixed (1:1) and the luminescent droplets were dropped onto the film. Protein bands were visualized using a gel imager (Bio-Rad Laboratories, Inc.). $\beta$-actin (cat. no. BA2305; Wuhan Boster Biological Technology, Ltd.) was used as the reference protein.

Enrichment analysis. Gene set enrichment analysis (GSEA) was performed to determine the correlation between the immune response in CC and MUC16 expression in patients with mutated and wild-type MUC16. Furthermore, genes were arranged from high to low according to their correlation with MUC16 expression. Genes and R values were determined and subsequently assessed via Kyoto Encyclopaedia of Genes and Genomes and Gene Ontology (GO) analyses within clusterProfiler v3.10.0 (http://www.bioconductor.org/packages/release/bioc/html/clusterProfiler.html) (21). Furthermore, single-sample (ss)GSEA was performed to calculate the score of immune response-associated GO terms (22). The gene set used for the ssGSEA was downloaded from the Molecular Signatures database (https://www. gsea-msigdb.org/gsea/msigdb/genesets.jsp).

Statistical analysis. Analysis of mutations was performed using R software version 2.5.0 (23). $\chi^{2}$ tests were used to analyze independent of data proportion for the proportion of MUC16 mutant/wild-type in CSCC/EAC. Student's unpaired t-test was used to determine differences in expression levels between two samples. The level of correlation was determined using Pearson correlation coefficient ( $R$ value) analysis. Kaplan-Meier survival curves and the log-rank test were used to determine the effects of MUC16 expression on patient survival, using GraphPad Prism software version 7 (GraphPad Software, Inc.). FPKM values, from the 20-80th percentiles were considered to classify the samples, and samples with the lowest log-rank P-values were selected. All graphs were generated using R software version 2.5.0 (RStudio, Inc.) or GraphPad Prism software version 7 (GraphPad Software, Inc.). Bar graph data are presented as the mean \pm SEM. 

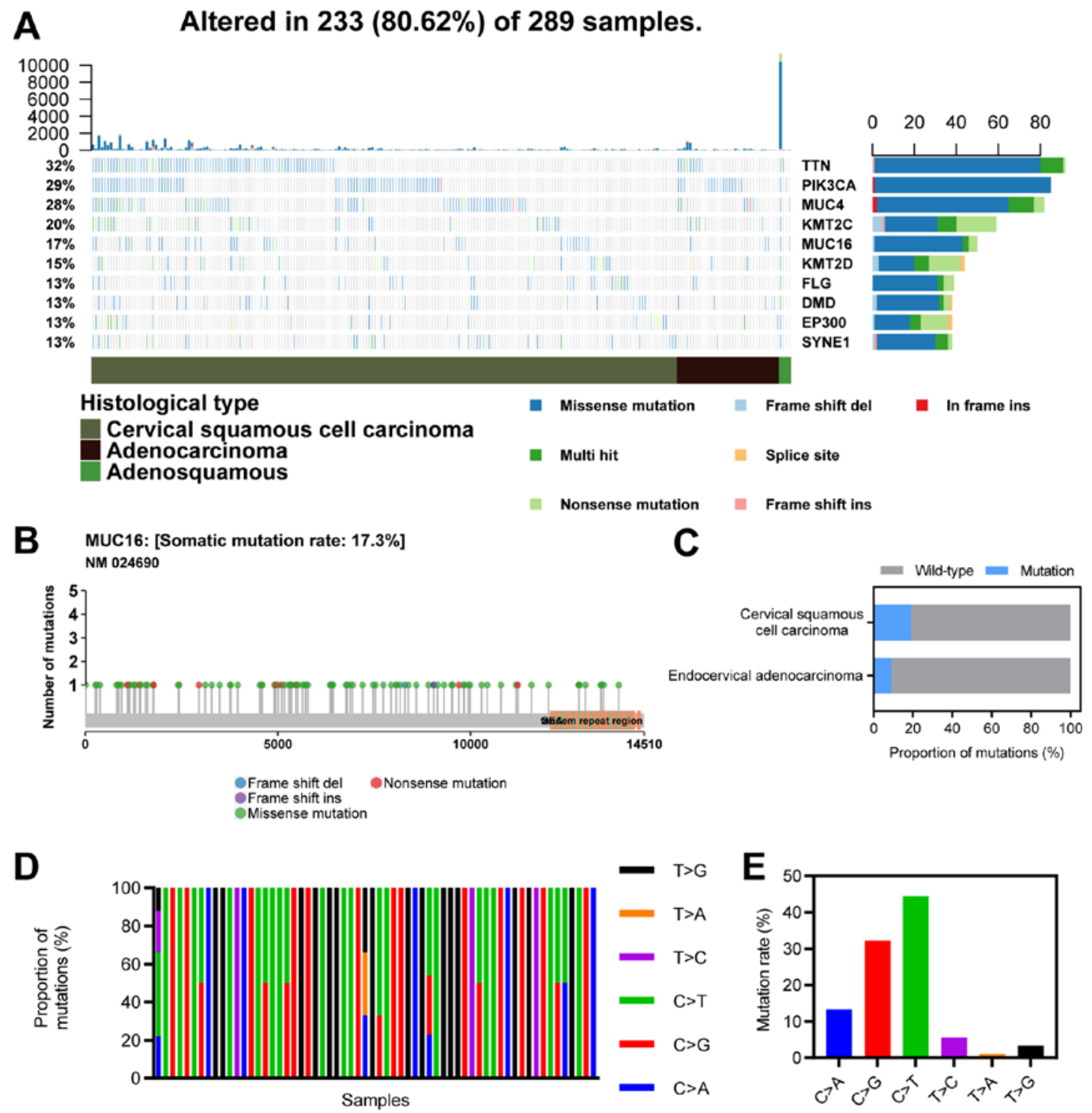

Figure 1. Landscape of MUC16 mutations in CC. (A) An OncoPlot of the top 10 mutated genes in CC samples from TCGA database. The upper bar plot indicates the number of genetic mutations/patient, while the bar plot on the right indicates the number of genetic mutations/gene. The CC pathology types and mutation types are represented as annotations at the bottom. (B) A lollipop plot of MUC16 mutations in CC samples from TCGA database. Amino acid axis labelled for domain. (C) Proportions of MUC16 mutations between endocervical adenocarcinoma and cervical squamous cell carcinoma. (D) Proportions of different types of single nucleotide variants of MUC16 in CC samples from TCGA database. (E) Numbers of different types of single nucleotide variants of MUC16 in CC samples from TCGA database. CC, cervical cancer; TCGA, The Cancer Genome Atlas; TTN, Titin; PIK3CA, phosphatidylinositol 3; MUC4, mucoprotein 4; KMT2C, histone lysine methyltransferase 2C; MUC16, mucoprotein 16; KMT2D, histone lysine methyltransferase 2D; FLG, filaggrin; DMD, duchenne muscular dystrophy; EP300, E1A binding protein p300; SYNE1, spectrin repeat-containing nuclear envelope protein 1.

\section{Results}

MUC16 is frequently mutated in CC. The top 10 mutated genes in CC included TTN (32\%), PIK3CA (29\%), MUC4 (28\%), KMT2C (20\%), MUC16 (17\%), KMT2D (15\%), FLG (13\%), DMD (13\%), EP300 (13\%) and SYNE1 (13\%; Fig. 1A). MUC16 ranked sixth among the most frequently mutated genes in $\mathrm{CC}$, with a mutational frequency of $17.3 \%$, and most mutations were missense mutations (Fig. 1B). The proportion of MUC16 mutations in CSCC (19\%) was greater than that in $\operatorname{EAC~}\left(9 \% ; \chi^{2}\right.$, $\mathrm{P}<0.05$; Fig. $1 \mathrm{C})$. Furthermore, $\mathrm{C}>\mathrm{G}$ and $\mathrm{C}>\mathrm{T}$ mutations were the major types of single nucleotide variants (Fig. 1D and E).

MUC16 expression in CC. MUC16 expression levels in different types of cancer were analyzed within the TCGA datasets (Fig. 2A). A total of three gynaecologic cancers, including ovarian, endometrial and $\mathrm{CC}$, ranked as having the highest MUC16 expression levels. Furthermore, the GEO dataset was used to assess MUC16 expression in tumour and normal samples, because TCGA lacks normal sample data for CC. The results demonstrated that MUC16 was significantly overexpressed in tumour samples compared with normal samples, according to the GSE9750 dataset (Fig. 2B, left panel). However, no significant difference was observed between mutated MUC16 and wild-type MUC16 in TCGA dataset (Fig. 2B, right panel). Subsequently, clinical human $\mathrm{CC}$ and adjacent normal samples were assessed to further investigate whether MUC16 is associated with CC. The results demonstrated that MUC16 protein expression was overexpressed in CC tissues compared with adjacent normal 


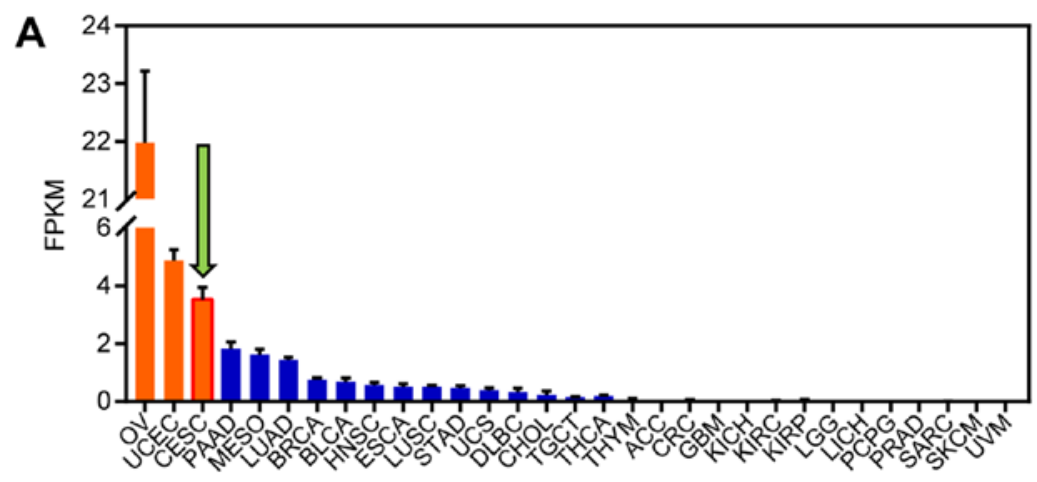

Cancer types
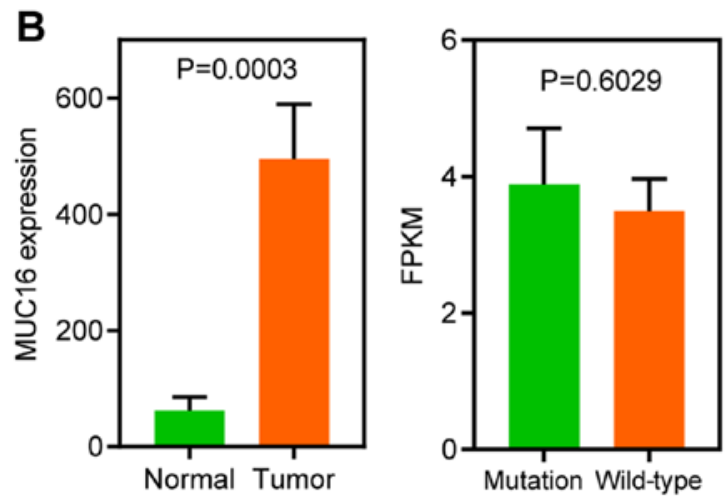

\section{C}

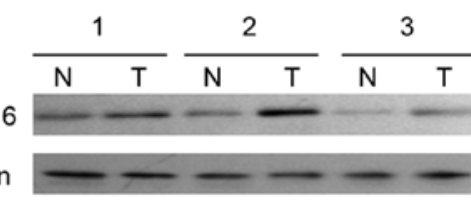

$\beta$-actin

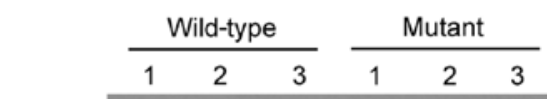

MUC16

$\beta$-actin

D
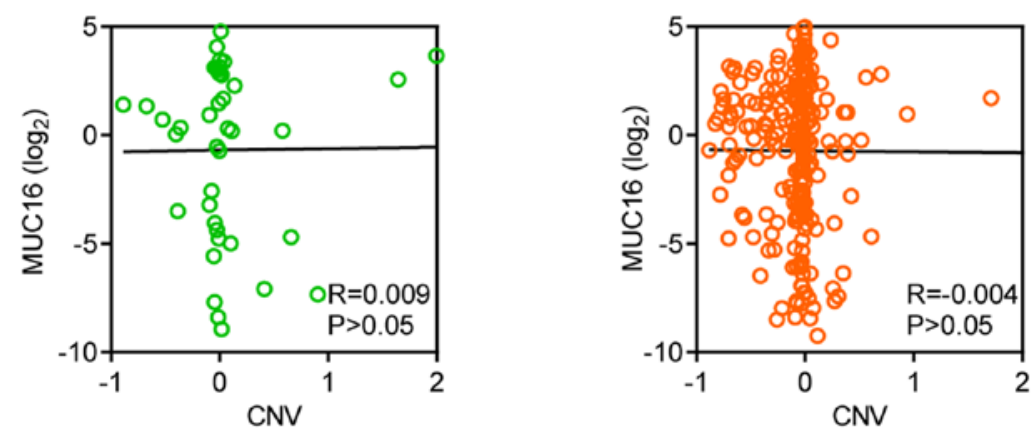

E
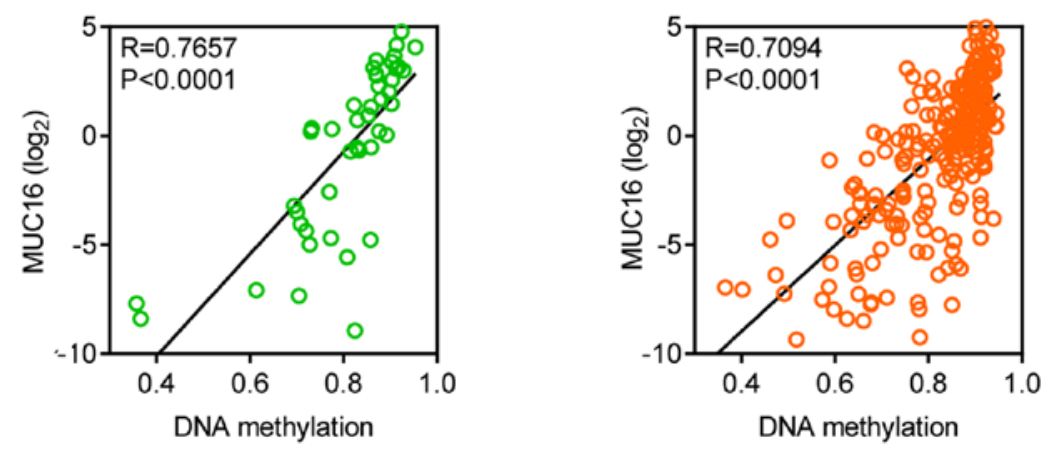

Figure 2. Landscape of MUC16 expression in CC. (A) Bar plot of FPKM for LINC00265 in different types of cancer from TCGA database. Data are presented as the mean \pm SEM. (B) Left panel: Bar plot depicting MUC16 expression in CC and normal samples from the GSE9750 dataset. Right panel: Expression levels of MUC16-mutated and MUC16-wild-type samples of CC from TCGA database. Data are presented as the mean \pm SEM. (C) MUC16 protein levels were determined via western blotting for normal and CC samples (top), and mutant and wild-type CC samples (bottom). (D) Association between CNV level and MUC16 expression in MUC16-mutated and MUC16-wild-type samples. (E) Association between DNA methylation level and MUC16 expression in MUC16-mutated and MUC16-wild-type samples. CC, cervical cancer; FPKM, fragments per kilobase million; TCGA, The Cancer Genome Atlas; SEM, standard error of the mean; $\mathrm{CNV}$, copy number variation.

tissues (Fig. 2C, top panel). However, no difference was observed between mutant and wild-type CC samples at the protein level (Fig. 2C, bottom panel). The CNV and DNA methylation of MUC16 was analyzed, using TCGA dataset, in order to determine the molecular mechanisms associated with MUC16 expression in CC. No correlation was observed between MUC16 expression and CNV in both the MUC16-mutated and the MUC16-wild-type groups (Fig. 2D). 
Conversely, DNA methylation was positively correlated with MUC16 expression in both the MUC16-mutated and the MUC16-wild-type groups (Fig. 2E). Taken together, the results demonstrated that MUC16 exhibited no difference between the MUC16-mutated and the MUC16-wild-type groups, although MUC16 was overexpressed in CC.

Overexpression of MUC16 is associated with favourable prognosis in MUC16 mutant patients with CC. For the purpose of evaluating the clinical significance of MUC16 in the survival of patients with $\mathrm{CC}$ and to determine the association between MUC16 mutational status and survival, MUC16 expression was assessed in $286 \mathrm{CC}$ clinical samples ( $\leq 4,000$ days of follow-up data) from TCGA for OS, DSS and PFS (24). Although OS is regarded as a vital endpoint, assessment of OS alone decreases the reliability of the results of a clinical study. When OS and DSS are used, longer follow-up is required. Therefore, PFS is used in many clinical trials as a composite of tumour progression and mortality (24). OS and DSS are associated with survival, while PFS is associated with recurrence. Overexpression of MUC16 was significantly associated with a longer OS time [hazard ratio $(\mathrm{HR})=0.156 ; \mathrm{P}=0.0399$ ], a longer $\mathrm{DSS}$ time $(\mathrm{HR}=0.277$; $\mathrm{P}=0.0374)$ and a longer $\mathrm{PFS}$ time $(\mathrm{HR}=0.271 ; \mathrm{P}=0.0205)$ in patients with CC and mutant MUC16 (Fig. 3A). However, overexpression of MUC16 was not associated with OS (HR=0.702; $\mathrm{P}=0.2122)$, DSS $(\mathrm{HR}=0.637 ; \mathrm{P}=0.1522)$ or $\mathrm{PFS}(\mathrm{HR}=1.570$; $\mathrm{P}=0.0926$ ) in patients with $\mathrm{CC}$ and wild-type MUC16 (Fig. 3B). Furthermore, overexpression of MUC16 had no significant effect on OS (HR=0.662; $\mathrm{P}=0.1082)$, DSS $(\mathrm{HR}=0.618 ; \mathrm{P}=0.0765)$ and PFS (HR=0.701; $\mathrm{P}=0.1660)$ in patients with CC (Fig. 3C). Taken together, these results suggested that overexpression of MUC16 may specifically predict a favourable prognosis in patients with $\mathrm{CC}$ and mutant MUC16.

Overexpression of MUC16 is associated with favourable prognosis in MUC16 mutant patients with CSCC. CSCC accounts for $70-80 \%$ of $\mathrm{CC}$ and antigen levels are associated with early-stage $\mathrm{CC}$ (4). In order to evaluate the clinical significance of MUC16 in terms of survival of patients with CSCC and to determine the association between MUC16 mutational status and survival, MUC16 expression was assessed in 240 CSCC clinical samples ( $\leq 4,000$ days of follow-up) from TCGA for OS, DSS and PFS. Overexpression of MUC16 was significantly associated with a longer OS time $(\mathrm{HR}=0 ; \mathrm{P}=0.0247)$, a longer DSS time $(\mathrm{HR}=0.247 ; \mathrm{P}=0.028)$ and a longer $\mathrm{PFS}$ time $(\mathrm{HR}=0.225$; $\mathrm{P}=0.019$ ) in patients with mutant MUC16 (Fig. 3D). However, overexpression of MUC16 was not associated with OS ( $\mathrm{HR}=1.39$; $\mathrm{P}=0.2477)$, DSS $(\mathrm{HR}=1.44 ; \mathrm{P}=0.293)$ and $\mathrm{PFS}(\mathrm{HR}=1.618$; $\mathrm{P}=0.1079$ ) in patients with wild-type MUC16 (Fig. 3E). Furthermore, overexpression of MUC16 had no significant effect on $\mathrm{OS}(\mathrm{HR}=0.266 ; \mathrm{P}=0.108)$, $\mathrm{DSS}(\mathrm{HR}=0.588 ; \mathrm{P}=0.0640)$ and $\mathrm{PFS}(\mathrm{HR}=0.701 ; \mathrm{P}=0.166)$ in patients with $\mathrm{CSCC}($ Fig. $3 \mathrm{~F})$. Taken together, these results suggested that overexpression of MUC16 may specifically predict a favourable prognosis in patients with CSCC and with mutant MUC16.

MUC16 mutations are involved in T cell-and B cell-related immune responses. MUC16 mutant patients with $\mathrm{CC}$ with MUC16 overexpression exhibited a longer OS, DSS and PFS time, which indicates that overexpression of MUC16 may be involved in the progression and tumorigenesis of MUC16-mutant CC. Previous studies have reported that MUC16 functions as a suppressor of the immune response (12-14). In order to determine whether overexpression of MUC16-mutant could decrease suppression of the immune response, GSEA was performed to identify immune response-associated gene sets in MUC16-mutant and MUC16-wild-type CC samples (Fig. 4A-C). Gene sets associated with $\mathrm{T}$ cell and $B$ cell activity were negatively enriched in CC samples with increased MUC16-wild-type expression. However, gene sets associated with $\mathrm{T}$ cell and $\mathrm{B}$ cell activity were not enriched in CC samples with increased MUC16-mutant expression. Taken together, these results suggested that overexpression of MUC16-mutant in patients with CC may help decrease the suppression of immune responses associated with $\mathrm{T}$ cells and $\mathrm{B}$ cells.

Immune responses are upregulated in early-stage patients with CC and MUC16 mutations. In order to investigate the association between immune responses, MUC16 mutation and early-stage $\mathrm{CC}$, an analysis of the differences between early-stage and stages I-IV CC samples was performed (Fig. 4D). ssGSEA was also performed to assess the gene sets associated with immune responses. The results demonstrated that immune responses associated with $\mathrm{T}$ cells and B cells were significantly upregulated in stages I/II samples with MUC16 mutations. However, no significant difference was observed between MUC16-wild-type samples of stages I/ II and stages III/IV. Furthermore, leukocyte-mediated immunity was significantly upregulated in stages I/II samples with MUC16 mutations. Taken together, these results indicated that immune responses were upregulated in patients with early-stage CC and with MUC16 mutations.

\section{Discussion}

$\mathrm{CC}$ is the fourth most common cancer in women worldwide (1). CC at the early stage can be treated with surgery or radiation (3). Furthermore, MUC16, which is one of the most frequently mutated genes in $\mathrm{CC}$, has been proven to serve as a tumour marker in different types of gynaecologic cancer, including CC (17). However, the association between MUC16 mutational status and prognosis of patients with CC remains unclear. To the best of our knowledge, the present study was the first to demonstrate the prognosis of patients with MUC16 mutations in CC. A recent study reported that MUC16 is also frequently mutated in endometrial cancer (16). The results of the present study demonstrated that the proportion of MUC16 mutations in CSCC is greater than that in EAC. Furthermore, MUC16 mutations were associated with early-stage CC. MUC16 expression was subsequently assessed in CC samples, and the results demonstrated that MUC16 was overexpressed in gynaecologic cancers. Although MUC16 overexpression was exhibited in $\mathrm{CC}$, there was no difference in expression between the MUC16-mutated and the MUC16-wild-type groups. Furthermore, differences in CNV or DNA methylation did not affect MUC16 expression in either the MUC16-mutated or MUC16-wild-type samples. These results suggested that the expression difference in MUC16 

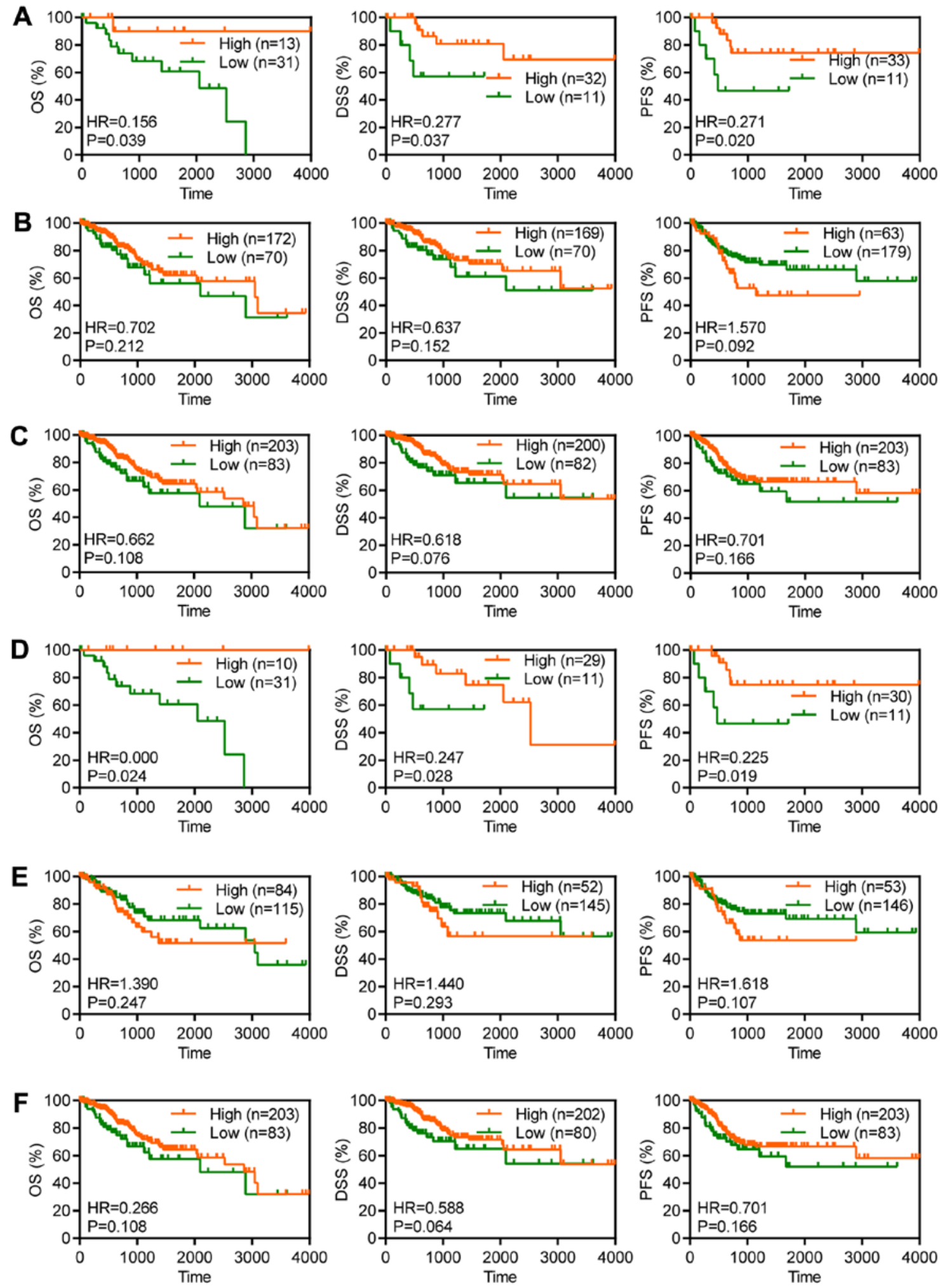

Figure 3. Overexpression of MUC16 was associated with favourable prognosis of patients with CC and mutant MUC16. Kaplan-Meier plots depicting the OS, DSS and PFS time in patients with (A) MUC16-mutant, (B) MUC16-wild-type and (C) all patients with CC from TCGA database. Kaplan-Meier plots depicting the OS, DSS and PFS time in patients with (D) MUC16-mutant, (E) MUC16-wild-type and (F) all patients with cervical squamous cell carcinoma from TCGA database. CC, cervical cancer; OS, overall survival; DSS, disease-specific survival; PFS, progression-free survival; TCGA, The Cancer Genome Atlas; HR, hazard ratio.

between MUC16-mutant and MUC16-wild-type samples may not have biological significance. However, it was subsequently observed that overexpression of mutant MUC16 may have biological significance. Overexpression of mutant
MUC16 specifically predicted favourable OS, DSS and PFS, whereas these associations were not present for wild-type MUC16 or MUC16 overall. Furthermore, overexpression of MUC16 specifically predicted favourable OS, DSS and 
A

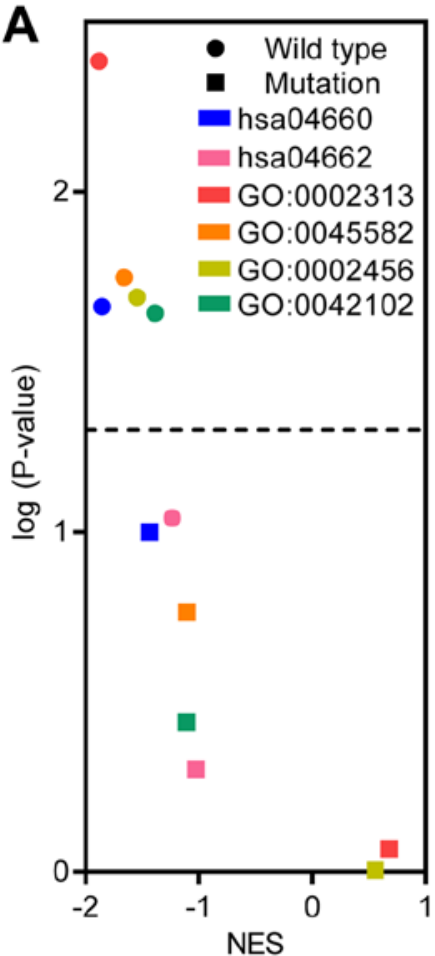

hsa04660 T cell receptor signaling pathway hsa04662 B cell receptor signaling pathway GO:0002313 Mature B cell differentiation involved inin GO:0042102 Positive regulation of $T$ cell proliferation

GO:0002456 T cell mediated immunity

GO:0045582 Positive regulation of $T$ cell differentiation
B GO:0002313 mature B cell differentiation involved in immune response
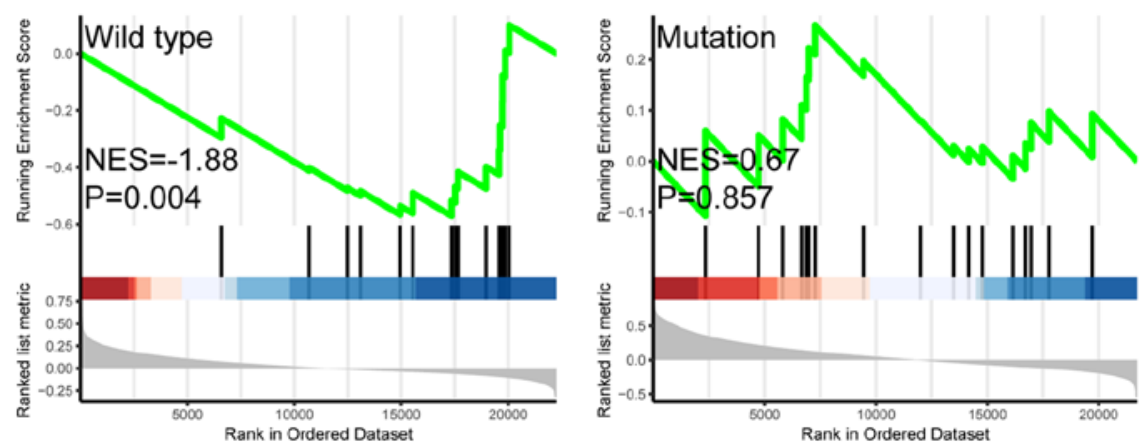

C GO:0002456 T cell mediated immunity

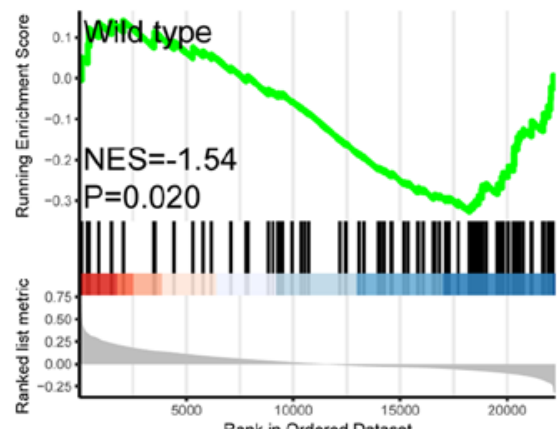

Rank in Ordered Dataset

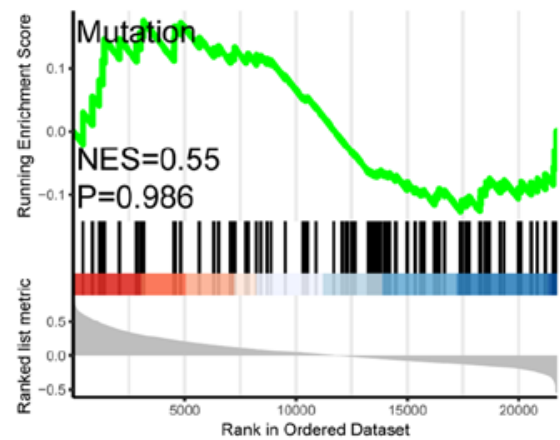

D
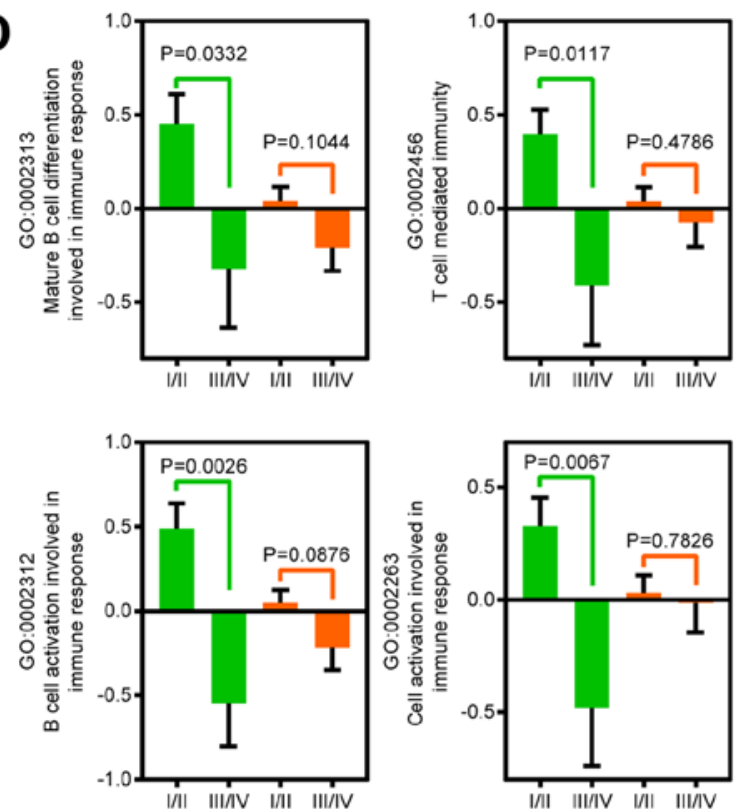
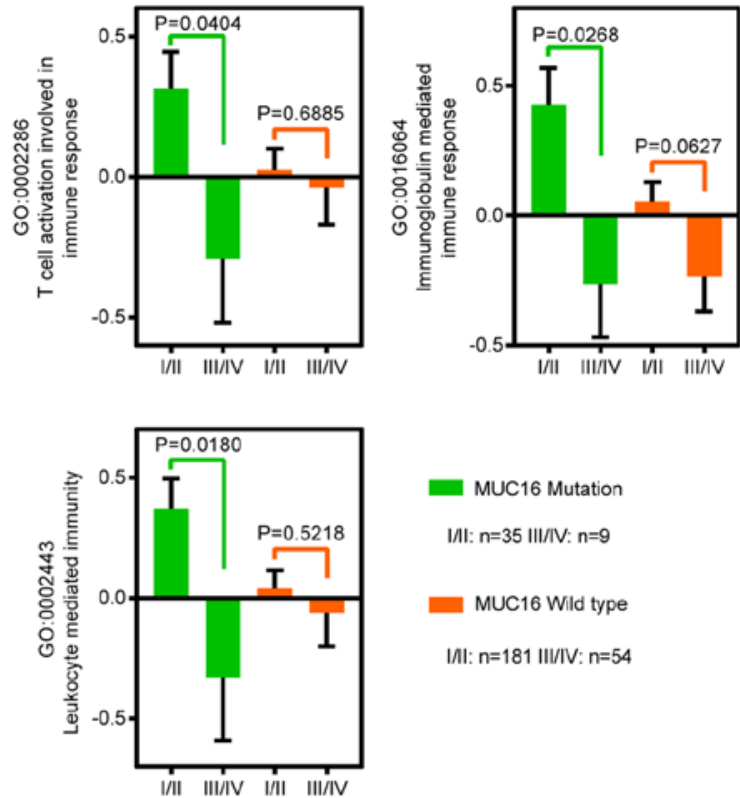

IMI: $n=181$ III/IV: $n=54$

Figure 4. MUC16 mutations were involved in immune responses. (A) Volcano plot depicting the NES and negative logarithmically transformed P-value of immune response-associated gene sets in MUC16-mutant and MUC16-wild-type CC samples from TCGA database. Enrichment of (B) B cell-associated and (C) T cell-associated gene sets with overexpression of MUC16, in MUC16-wild-type and MUC16-mutant CC samples from TCGA database. (D) Bar plots depicting the activity level of multiple immune responses in different disease stages between MUC16-mutant and MUC16-wild-type samples of CC from TCGA database. Data are presented as the mean \pm standard error of the mean. NES, normalized enrichment score; CC, cervical cancer; TCGA, The Cancer Genome Atlas.

PFS in patients with MUC16-mutant CSCC. Therefore, the results suggested that overexpression of MUC16 may predict favourable prognosis in both patients with MUC16-mutant $\mathrm{CC}$ and patients with MUC16-mutant CSCC. MUC16 served as an essential prognostic biomarker in $\mathrm{CC}$, particularly in terms of clinical survival associated with early-stage disease.
The immune response is of great significance for the development and progression of CC (25-27). Increasing evidence demonstrates that gene mutations are associated with cancer progression, immune response and clinical outcomes in patients with early-stage disease (28-30). Furthermore, it has been demonstrated that MUC16 interacts actively with the 
immune response (12-14). Previous studies have reported that MUC16 mutations are closely associated with superior survival outcomes and the immune response in gastric and endometrial cancers $(12-14,16)$. However, very few studies have investigated the effects of MUC16 mutations on the immune response in patients with CC. Previous studies have demonstrated that MUC16 acts as a suppressor of immune response (12-14,16). In the present study, GSEA demonstrated that negative enrichment of immune response signatures, such as immune responses associated with $\mathrm{T}$ cells and $\mathrm{B}$ cells, was observed with overexpression of MUC16 in the MUC16-wild-type CC samples. However, the signatures associated with $\mathrm{T}$ cell and $B$ cell activity revealed no enrichment with overexpression of MUC16 in the MUC16-mutant CC samples. The present study demonstrated that MUC16 mutations are associated with early-stage $\mathrm{CC}$. Thus, the present study investigated the immune responses associated with $\mathrm{T}$ cells and $\mathrm{B}$ cells during different stages of disease in patients with MUC16 mutations. Immune responses were upregulated in stages I/II of MUC16 patients with $\mathrm{CC}$ patients, but not in MUC16-wild-type patients with $\mathrm{CC}$ patients.

In conclusion, the results from the present study suggested that overexpression of MUC16 may specifically predict favourable survival and prognosis in patients with MUC16-mutant CC. Furthermore, MUC16 mutational status was associated with the immune response in $\mathrm{CC}$, particularly in patients at early stage of disease. MUC16 mutations are a key marker in immunotherapy for CC patients. MUC16 overexpression is not only significantly associated with the prognosis of MUC16-mutant patients with $\mathrm{CC}$, but also lead to the enrichment of associated immune activity, which can be used to identify which patients may benefit from immunotherapy in CC. Taken together, the findings from the present study provided novel and promising approaches for immunotherapy in $\mathrm{CC}$; however, further studies are required to investigate these methods.

\section{Acknowledgements}

Not applicable.

\section{Funding}

No funding was received.

\section{Availability of data and materials}

The datasets generated and analyzed during the current study are available in the TCGA dataset [https://portal.gdc.cancer. gov/] and GSE9750 dataset [https://www.ncbi.nlm.nih.gov/ geo/query/acc.cgi?acc=GSE9750].

\section{Authors' contributions}

HW and CY conceptualized the study, designed the research and performed the bioinformatics analysis. HW performed the experiments. HW and HY analyzed and interpreted the data. HW wrote and edited the manuscript, and HY supervised the project. All authors read and approved the final manuscript.

\section{Ethics approval and consent to participate}

All experimental procedures were approved by the Ethics Committee of The First College of Clinical Medical Science, China Three Gorges University (Yichang, China). Written informed consent was provided by all patients prior to the study.

\section{Patient consent for publication}

Not applicable.

\section{Competing interests}

The authors declare that there have no competing interests.

\section{References}

1. Bray F, Ferlay J, Soerjomataram I, Siegel RL, Torre LA and Jemal A: Global cancer statistics 2018: GLOBOCAN estimates of incidence and mortality worldwide for 36 cancers in 185 countries. CA Cancer J Clin 68: 394-424, 2018.

2. Wong FL and Miller JW: Centers for Disease Control and Prevention's National Breast and Cervical Cancer Early Detection Program: Increasing Access to Screening. J Womens Health (Larchmt) 28: 427-431, 2019.

3. Cancer Genome Atlas Research Network; Albert Einstein College of Medicine; Analytical Biological Services; Integrated genomic and molecular characterization of cervical cancer. Nature 543: 378-384, 2017.

4. MarthC,LandoniF,MahnerS,McCormackM,Gonzalez-Martin A, Colombo $\mathrm{N}$ and Committee EG: Cervical cancer: ESMO clinical practice guidelines for diagnosis, treatment and follow-up. Ann Oncol 28: iv72-iv83, 2017.

5. Reesink-Peters N, van der Velden J, Ten Hoor KA, Boezen HM, de Vries EG, Schilthuis MS, Mourits MJ, Nijman HW, Aalders JG, Hollema H, et al: Preoperative serum squamous cell carcinoma antigen levels in clinical decision making for patients with early-stage cervical cancer. J Clin Oncol 23: 1455-1462, 2005.

6. Jonckheere $\mathrm{N}$ and Van Seuningen I: Integrative analysis of the cancer genome atlas and cancer cell lines encyclopedia large-scale genomic databases: MUC4/MUC16/MUC20 signature is associated with poor survival in human carcinomas. J Transl Med 16: 259, 2018

7. Hollingsworth MA and Swanson BJ: Mucins in cancer: Protection and control of the cell surface. Nat Rev Cancer 4: 45-60, 2004.

8. Rao CV, Janakiram NB and Mohammed A: Molecular pathways: Mucins and drug delivery in cancer. Clin Cancer Res 23: 1373-1378, 2017.

9. Das S and Batra SK: Understanding the unique attributes of MUC16 (CA125): Potential implications in targeted therapy. Cancer Res 75: 4669-4674, 2015.

10. Lakshmanan I, Salfity S, Seshacharyulu P, Rachagani S, Thomas A, Das S, Majhi PD, Nimmakayala RK, Vengoji R, Lele SM, et al: MUC16 regulates TSPYL5 for lung cancer cell growth and chemoresistance by suppressing p53. Clin Cancer Res 23: 3906-3917, 2017.

11. Streppel MM, Vincent A, Mukherjee R, Campbell NR, Chen SH, Konstantopoulos K, Goggins MG, Van Seuningen I, Maitra A and Montgomery EA: Mucin 16 (cancer antigen 125) expression in human tissues and cell lines and correlation with clinical outcome in adenocarcinomas of the pancreas, esophagus, stomach, and colon. Hum Pathol 43: 1755-1763, 2012.

12. Gubbels JA, Felder M, Horibata S, Belisle JA, Kapur A, Holden H, Petrie S, Migneault M, Rancourt C, Connor JP, et al: MUC16 provides immune protection by inhibiting synapse formation between NK and ovarian tumor cells. Mol Cancer 9: 11, 2010.

13. Patankar MS, Jing Y, Morrison JC, Belisle JA, Lattanzio FA, Deng Y, Wong NK, Morris HR, Dell A and Clark GF: Potent suppression of natural killer cell response mediated by the ovarian tumor marker CA125. Gynecol Oncol 99: 704-713, 2005.

14. Fan K, Yang C, Fan Z, Huang Q, Zhang Y, Cheng H, Jin K, Lu Y, Wang Z, Luo G, et al: MUC16 C terminal-induced secretion of tumor-derived IL-6 contributes to tumor-associated Treg enrichment in pancreatic cancer. Cancer Lett 418: 167-175, 2018. 
15. Li X, Pasche B, Zhang W and Chen K: Association of MUC16 mutation with tumor mutation load and outcomes in patients with gastric cancer. JAMA Oncol 4: 1691-1698, 2018.

16. Hu J and Sun J: MUC16 mutations improve patients' prognosis by enhancing the infiltration and antitumor immunity of cytotoxic $\mathrm{T}$ lymphocytes in the endometrial cancer microenvironment. Oncoimmunology 7: e1487914, 2018.

17. Aggarwal $P$ and Kehoe S: Serum tumour markers in gynaecological cancers. Maturitas 67: 46-53, 2010.

18. Lee YY, Kim TJ, Kim JY, Choi CH, Do IG, Song SY, Sohn I, Jung SH, Bae DS, Lee JW, et al: Genetic profiling to predict recurrence of early cervical cancer. Gynecol Oncol 131: 650-654, 2013.

19. Scotto L, Narayan G, Nandula SV, Arias-Pulido H, Subramaniyam S, Schneider A, Kaufmann AM, Wright JD, Pothuri B, Mansukhani M, et al: Identification of copy number gain and overexpressed genes on chromosome arm $20 \mathrm{q}$ by an integrative genomic approach in cervical cancer: Potential role in progression. Genes Chromosomes Cancer 47: 755-765, 2008.

20. Liu J, Lichtenberg T, Hoadley KA, Poisson LM, Lazar AJ, Cherniack AD, Kovatich AJ, Benz CC, Levine DA, Lee AV, et al: An integrated TCGA pan-cancer clinical data resource to drive high-quality survival outcome analytics. Cell 173: 400-416.e11, 2018.

21. Yu G, Wang LG, Han Y and He QY: clusterProfiler: An R package for comparing biological themes among gene clusters. OMICS 16: 284-287, 2012.

22. Hänzelmann S, Castelo R and Guinney J: GSVA: Gene set variation analysis for microarray and RNA-seq data. BMC Bioinformatics 14: 7, 2013

23. Mayakonda A, Lin DC, Assenov Y, Plass C and Koeffler HP: Maftools: Efficient and comprehensive analysis of somatic variants in cancer. Genome Res 28: 1747-1756, 2018.

24. Johnson KR, Liauw W and Lassere MN: Evaluating surrogacy metrics and investigating approval decisions of progression-free survival (PFS) in metastatic renal cell cancer: A systematic review. Ann Oncol 26: 485-496, 2015.
25. Punt S, Houwing-Duistermaat JJ, Schulkens IA, Thijssen VL, Osse EM, de Kroon CD, Griffioen AW, Fleuren GJ, Gorter A and Jordanova ES: Correlations between immune response and vascularization qRT-PCR gene expression clusters in squamous cervical cancer. Mol Cancer 14: 71, 2015.

26. Heeren AM, Koster BD, Samuels S, Ferns DM, Chondronasiou D, Kenter GG, Jordanova ES and de Gruijl TD: High and interrelated rates of $\mathrm{PD}-\mathrm{L} 1^{+} \mathrm{CD} 14^{+}$antigen-presenting cells and regulatory $\mathrm{T}$ cells mark the microenvironment of metastatic lymph nodes from patients with cervical cancer. Cancer Immunol Res 3: 48-58, 2015.

27. Cui JH, Lin KR, Yuan SH, Jin YB, Chen XP, Su XK, Jiang J, Pan YM, Mao SL, Mao XF, et al: TCR repertoire as a novel indicator for immune monitoring and prognosis assessment of patients with cervical cancer. Front Immunol 9: 2729, 2018

28. Mehnert JM, Panda A, Zhong H, Hirshfield K, Damare S, Lane K, Sokol L, Stein MN, Rodriguez-Rodriquez L, Kaufman HL, et al: Immune activation and response to pembrolizumab in POLE-mutant endometrial cancer. J Clin Invest 126: 2334-2340, 2016.

29. Choi M, Kadara H, Zhang J, Parra ER, Rodriguez-Canales J, Gaffney SG, Zhao Z, Behrens C, Fujimoto J, Chow C, et al: Mutation profiles in early-stage lung squamous cell carcinoma with clinical follow-up and correlation with markers of immune function. Ann Oncol 28: 83-89, 2017.

30. McFarland CD, Yaglom JA, Wojtkowiak JW, Scott JG, Morse DL, Sherman MY and Mirny LA: The damaging effect of passenger mutations on cancer progression. Cancer Res 77: 4763-4772, 2017.

This work is licensed under a Creative Commons Attribution-NonCommercial-NoDerivatives 4.0 International (CC BY-NC-ND 4.0) License. 